\title{
New ideas and research directions for multi-level and multi-dimensional dynamic systems
}

\author{
Guoxin Yang* \\ Shanghai Jiao Tong University \\ Carl Yang ${ }^{\dagger}$ \\ University of Illinois, Urbana Champaign
}

(Dated: September 13, 2020)

\begin{abstract}
Energieelement is the most microscopic unit of human understanding of the universe. It consists of energieelement quantum vortex motion to form the dynamic system of different levels and forms, and then forms the universe in various shapes and forms. The multi-level and multi-dimensional dynamic system is the basic motion law of everything in the universe from macro to micro. Through the operation of solid model, mathematical description and computer simulation of multi-level and multi-dimensional dynamic systems, the operation law of everything in the universe from macro to micro is revealed, and various natural phenomena and scientific observations are explained. At the same time, effective approaches and methods are provided for the exploration and understanding of unsolved physical phenomena or problems [1].
\end{abstract}

\section{INTRODUCTION}

The universe and all things move, interact and change in a multi-level and multi-dimensional dynamic system. On the macro level, from galaxy groups, galaxies, stars and planets to the various and ever-changing universe, all are dynamic systems at different levels. On the micro level, from molecules, atoms, protons and neutrons to electrons, neutrinos, quarks, gluons and other basic particles to energieelements, all are dynamic systems of different levels.

\section{THE BASIC CHARACTERISTICS OF THE MULTI-LEVEL AND MULTI-DIMENSIONAL DYNAMIC SYSTEM}

A system with the same laws of motion, composed of a particular energy and substance, is a dynamic system. Any dynamic system has the following characteristics:

1. The same dynamic system includes multiple individuals. For example, the solar system includes the sun and all the planets orbiting it; Each atom contains all the subatomic particles that make up the atom.

2. Each member of the same dynamic system follows the same system operation law (or mode of motion). For example, all stars and planets in the Milky Way follow the spiral arms of the Milky Way; The quarks and gluons that make up the proton follow the law of rotation and motion of the proton.

\footnotetext{
*yangguoxinsc@abchina.com

$\dagger$ jiyang3@illinois.edu
}

3. Any dynamic system has specific spatial and temporal characteristics such as space, time and motion direction. The spatial and temporal characteristics of different dynamic systems, especially those at different levels, are significantly different.

4. The different levels of subsystems contained in any dynamic system, from micro to macro, can be defined as dynamic systems with different multiplicity. For example, elementary particles, subatomic particles, atoms, molecules, planets, stars, galaxies, groups of galaxies, etc., can be divided into multiple dynamic systems.

5. The dynamic system at any level rotates periodically.

6. In multiple dynamic systems, the results of observing the motion trajectory of the same dynamic system from different levels of dynamic systems are completely different. For example, if you look at the orbits of the planets in the solar system, you see that they rotate statically relative to the sun. But if you look at the orbits of the planets in our solar system from the Milky Way galaxy, you see them in a spiral motion around the sun.

7. There is no isolated or single dynamic system in the universe. Different levels or different dynamic systems interact through energy radiation or exchange. High-energy radiation, such as photons, neutrinos, and other cosmic rays, can travel through, or away from, several dynamic systems. The interaction between dynamic systems is also used as a dynamic mechanism to generate or maintain rotational motion and dynamic stability of the dynamic system. 


\section{THE MOTION LAW OF THE MULTI-LEVEL AND MULTI-DIMENSIONAL DYNAMIC SYSTEM}

From the macro to the micro level, all the universe and everything are moving and changing in different multilevel and multi-dimensional dynamic systems. These different levels, various forms and ever-changing universe and everything have the basic structure of the multi-level and multi-dimensional dynamic system and follow the basic motion law of the multi-level and multi-dimensional dynamic system.

1. The dynamic system of same level or with the same heaviness does not exist in isolation, but is composed of many similar dynamic systems combined in a specific way and following the same law of motion.

2. If the matter or energy of the universe is divided from macro to micro into different levels or multiplies from top to bottom, the dynamic system of the next level follows the motion law of the dynamic system of the next level, or the dynamic system of the next level follows the motion law of the next level. If a dynamic system of certain heaviness is composed of several dynamic systems of less heaviness, then any of the dynamic systems of less heaviness that make up the dynamic system of certain heaviness obey the motion law of this dynamic power system. For example, the subatomic particles and elementary particles that make up atoms obey the laws of atomic motion; The atoms that make up a molecule obey its laws of motion; The molecules that make up plants and animals obey the laws of movement of individual plants and animals; The planets of the solar system obey the motion law of the solar system; The Milky Way's stars and interstellar matter obey the laws of the Milky Way's motion and so on.

3. Dynamic systems of different heaviness have different or unique spatial and temporal characteristics, which are mainly manifested in different size of the dynamic system of certain heaviness, threedimensional space direction, rotation speed (period or frequency) of the dynamic system, etc.

\section{DESCRIPTION OF MOTION LAW OF THE MULTI-LEVEL AND MULTI-DIMENSIONAL DYNAMIC SYSTEM}

No matter from the macroscopic galaxies of the universe to everyday things, or from the microscopic world of molecules to elementary particles, multi-level and multidimensional dynamic systems are the basic structures and laws of motion. For example, elementary particles obey the rotational motion of protons or neutrons while rotating themselves; A proton or neutron obeys the rotational motion of an atom while rotating itself; The earth rotates and obeys the rotation of the solar system; The solar system rotates in accordance with the rotation of the Milky Way, and so on. Now a simplified multi-level and multi-dimensional dynamic system is described by using the five-fold dynamic system.

\section{A. multi-level and multi-dimensional dynamic system model}

A simplified five-fold multi-level and multi-dimensional dynamic system entity model is shown in Fig. 1. Specifically, the 5-fold multi-level and multi-dimensional dynamic system is modeled by five rotating circles with different radius, different directions of rotation, and different speeds of rotation. Their motion has a dependent property, that is, the next heaviness circle has the motion law of the previous heaviness circle (rotation direction and speed), and the smallest heaviness circle has the motion law of the above four heaviness circles at the same time, see the position and motion track of the points marked in white in the figure.

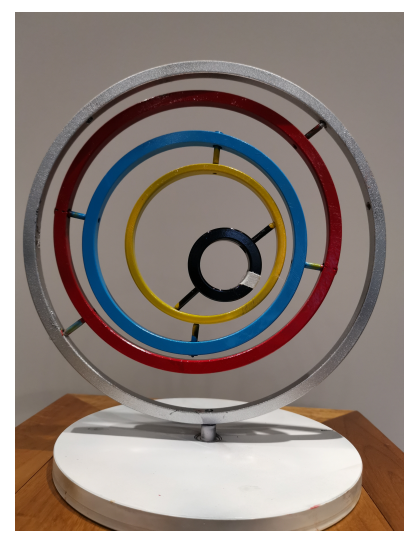

(a)Model in static

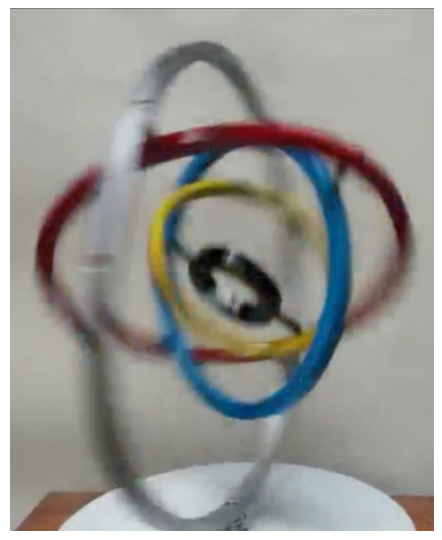

(b)Model in motion
FIG. 1. Real-world multi-level and multi-dimensional dynamic system model.

\section{B. Computer simulation of a multi-level and multi-dimensional dynamic system}

The multi-level and multi-dimensional dynamic system is simulated by computer, and a rotation model of a fivefold sphere is established. A grey spinning sphere has radius $R_{1}$ and period $T_{1}$; The grey sphere has a red spinning sphere of radius $R_{2}$ and period $T_{2}$; The red sphere has a blue spinning sphere with a radius of $R_{3}$ and a period of $T_{3}$; The blue ball has a yellow spinning ball with radius $R_{4}$ and period $T_{4}$. The yellow sphere has a black spinning sphere of radius $R_{5}$ and period $T_{5}$. The centers of the 5 -fold multiple balls do not overlap. The 5 -fold 


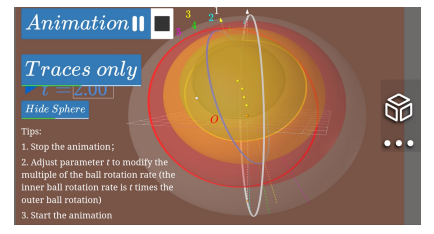

(a)

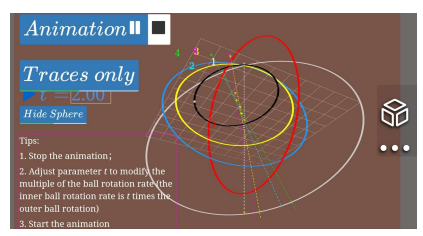

(b)

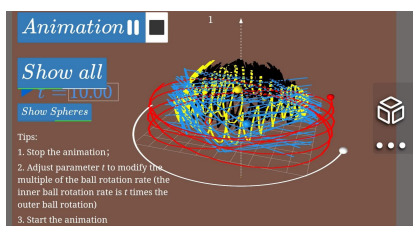

(c)

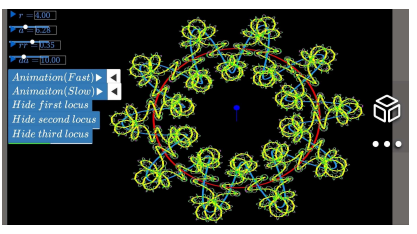

(d)

FIG. 2. Computer simulated multi-level and multi-dimensional dynamic system model.

multiple balls rotates 15 to 45 degrees apart from each other as it begins to spin. See Fig. 2 (a) for the operation effect of the rotating motion model of the 5 -fold multiple spheres [2].

The computer simulation of the rotation motion of the 5 -fold circles when the 5-fold balls are hidden is shown in Fig. 2 (b) [2]. Each point is marked on the 5-fold circles. See Fig. 2 (c) for the five-fold track of the rotation motion of the five-fold circles [3]. The multiple trajectories of the rotation motion of the computer-simulated multiple power systems are shown in Fig. 2 (d) [4].

\section{The mathematical description of a multi-level and multi-dimensional dynamic system}

Suppose a dynamic system with $n$ layers, the outermost layer is 1 , and the smallest (lowest) layer is $n$. In the mathematical description, the center of the rotating motion dynamical system of the first layer is taken as the origin of coordinates, and the plane rotating through the origin is taken as the base plane. On the base plane are the $X$-axis and $Y$-axis; the $Z$-axis is perpendicular to the base plane, and the $X Y Z$ axis constitutes the three-dimensional space coordinate system of the multidimensional dynamic system. The dynamic system $\left(n_{i}\right)$ of each layer takes the orbital line of the dynamic system $\left(n_{i-1}\right)$ of the next layer as the origin, and the initial angle (also called inclination angle) between the rotating surface and the foundation plane of the first dynamical system is $\theta_{i}$. The rotation radius of the $i$ th dynamical system is the distance $R_{i}$ between its origin and the origin of the $(i+1)$ th dynamic system, the rotational motion velocity (or period) of the dynamic system of $i$ th layer is $T_{i}$. Thus, the motion trajectory of each dynamic system is shown in Figure 3.

The trajectory of the first dynamic system is in the plane $X Y$ and passing through the origin $O$, with radius $R_{1}$, inclination 0 and operating period $T_{1}$. The motion trajectory of the first dynamical system is $\xi_{1}\left(R_{1}, \theta_{1}, T_{1} \mid 0\right)$, that is $\xi_{1}\left(R_{1}, 0, T_{1} \mid 0\right)$.

When the second dynamic system rotates along the first orbit, it also rotates itself. Its rotation surface has a dip angle of $\theta_{2}$ with $X O Y$ at the beginning, the radius of rotation motion is $R_{2}$, and the period of rotation motion is $T_{2}$. Then the motion trajectory of the second power system is $\xi_{2}\left(R_{2}, \theta_{2}, T_{2} \mid \xi_{1}\right)$. Thus the trajectories of the first and second dynamic systems are iteratively merged into the second dynamic system.

By the same notions, the dynamic system of $i$ th layer rotates along the trajectory line of the dynamic system of the $(i-1)$ th layer, and the radius of its rotation surface is $R_{i}$, the rotation surface has a dip angle of $\theta_{i}$ with $X O Y$ at the beginning, and the rotation motion period is $T_{i}$. Then the operating orbit of the dynamical system of the $i$ th layer is $\xi_{i}\left(R_{1}, \theta_{i}, T_{i} \mid \xi_{i-1}\right)$. Here $\xi_{i-1}$ means the original of the dynamic system of the $i$ th layer is rotating along the trajectory line of the dynamic system of the $(i-1)$ th layer.

If the multi-level and multi-dimensional dynamic system has $n$ layers, the trajectory line of the lowest layer (or internal minimum layer is $n$ ) is $\xi_{n}\left(R_{n}, \theta_{n}, T_{n} \mid \xi_{n-1}\right)$, which iteratively merges the trajectory lines of all dynamic systems of $n$ layers into the trajectory line of the $n$th layer.

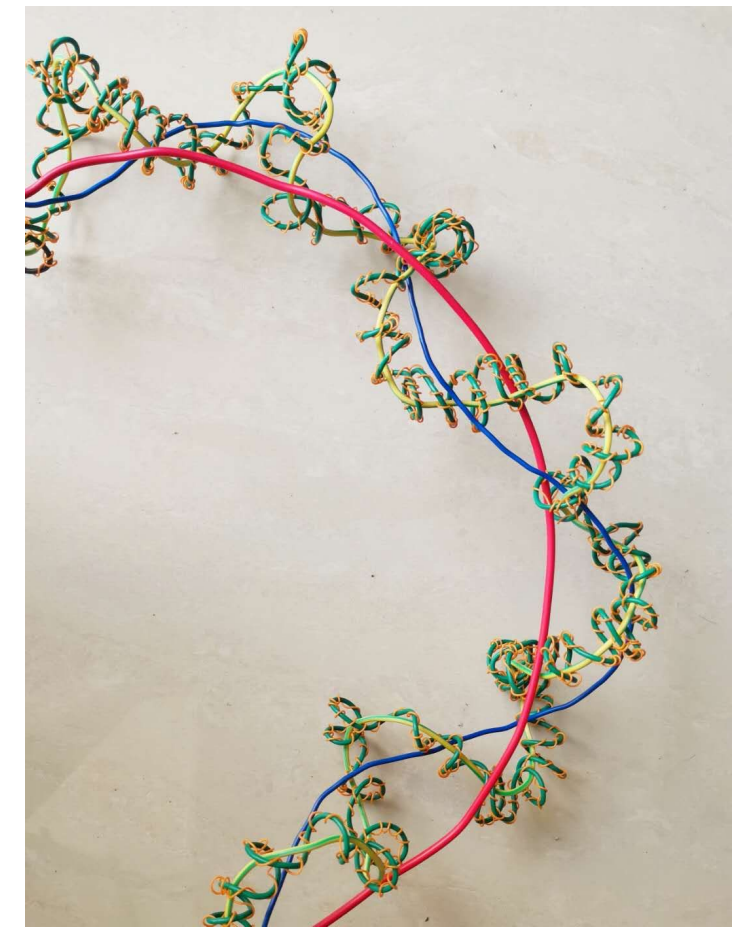

FIG. 3. The motion trajectory of the dynamic system. 


\section{THE MULTI-LEVEL AND MULTI-DIMENSIONAL DYNAMIC SYSTEM IS THE BASIC STRUCTURE AND MOTION LAW OF THE UNIVERSE.}

The system can be used to recognize and understand various natural phenomena and physical laws.

\section{A. Explanations}

\section{Higher dimensional space-time and multiverse}

It is easy to know and understand higher dimensional space-time and multiverse [5] by replacing them with multi-level and multi-dimensional dynamic systems. From the point of view of multi-level and multiple-level dynamic systems, from the Milky Way galaxy, solar system, earth, atom, proton (neutron) to elementary particle are all dynamic systems of different levels, and are also inertial motion systems of different levels. If each dynamic system is defined as one space-time dimension, then a multi-level and multi-dimensional spacetime system, also called a multi-universe, is formed from macro to micro. The space-time dimension composed of multiple dynamic systems is a higher-dimensional spacetime system beyond the current four-dimensional spacetime. Phenomena and problems beyond four dimensional space-time systems can be scientifically understood and explained in higher dimensional space-time systems.

\section{Spatiotemporal curvature [6] is represented in multi-level and multi-dimensional dynamic systems}

Because each dimension of space-time (each rotating dynamic system) has different size, different rotation direction, and different motion time (period or frequency), they determine different space-time characteristics. Therefore, the space-time formed by the dynamic system with different multiplicity has different spacetime characteristics. The space-time formed by different multi-level and multi-dimensional dynamic systems also has different space-time characteristics. The spacetime of these different characteristics is the difference of space-time in different regions of the universe, and the difference of space-time in different regions is manifested as the curvature of space-time. As it travels through the universe, the radiation of light will pass through many different dynamic systems, that is, through space-time of many different properties, and as it passes through spacetime of these different properties, it will be subjected to its effects and produce different changes. As the light passes through a powerful dynamic system, for example, passing through a layer of water vapor, passing through a prism and so on, it will change in a different way. When radiated energy, such as light, passes through a dynamic system with multi-levels or through several dynamic systems, they will act on it and leave a corresponding trace.

\section{Multi-dimensional spatial and temporal characteristics of multi-level and multi-dimensional dynamic systems}

From the macro perspective, everyday things are the first dynamic system, including animals, plants, atmosphere, oceans, etc. For example, the movement changes of the atmosphere show the characteristics of space-time, such as wind and waves, rain or shine and snow. The rotation of the earth is the second dynamic system, and its motion is characterized by 24-hour period, alternation of days and nights and other spatiotemporal characteristics. The earth's revolution (that is, the rotation in the solar system) is the third dynamic system, and its motion changes show the spatiotemporal characteristics such as the 365-day cycle and the alternation of spring, summer, autumn and winter seasons. The motion of the solar system around the center of the Milky Way galaxy is the fourth dynamic system, and its motion in the Milky Way galaxy is characterized by the period of 226 million years and the climate change over tens of millions of years [1]. The motion of the Milky Way galaxy in the galaxy cluster forms the fifth dynamic system [7]. Too little is known or experienced of the temporal and spatial properties of the fifth dynamic system, just like the extinction of the dinosaurs, human beings are only 5 million years old now. Hundreds of millions of years later, human beings may disappear like the dinosaurs. In this way, human beings will never understand or experience the spatiotemporal characteristics of the fifth dynamic system.

From the microscopic perspective, the molecules of the object are the first dynamic system. Molecules determine the basic properties of an object, such as the density (specific gravity), hardness, temperature, solid, liquid, gas and other different states. These basic properties are the space-time properties of the molecular level. The atom is the second dynamic system. The periodic nature of its motion (as listed in the periodic table) is the spacetime nature of the atomic level. Subatomic particles such as protons and neutrons are the third dynamic system. Their motion period and motion mode are the spacetime characteristics of the subatomic level. Electrons, quarks and bosons are the fourth dynamic system, and their motion period and mode (such as quantum properties) are the space-time properties of elementary particles. The energieelement of elementary particles is the fifth dynamic system [8]. At present, the structure and motion law of this hierarchical dynamical system are not clear. The world's most powerful accelerator, the Large Hadron Collider (LHC) at CERN, has an energy scale of $10^{13}$ electron volts. To detect the structure and motion of energy quanta requires a high energy collider of $10^{24}$ to $10^{28}$ electron volts, it would require building colliders that are between a trillion kilometers and 1,000 lightyears long, which would be too difficult for humans to 
handle. Humans may have disappeared long before they were finished, and in that sense it is impossible to detect the structure and motion of a quantum.

\section{Quantum effects [9] being the basic law of multi-level and multi-dimensional dynamic systems}

The uncertainty (randomness and superposition) of quantum motion is the essential property of quantum motion in multi-level and multi-dimensional dynamic systems. There are multiple dynamic systems on the quantum. Since each dynamic system has different space-time characteristics (size, direction, and period), the quantum motion follows the motion characteristics (law) of each dynamic system on the quantum. Since quantum simultaneously has the motion characteristics of multi-level and multi-dimensional dynamic systems (magnitude, direction, period), the motion trajectory of quantum must be the superposition and combination of the motion trajectories of the multi-level and multi-dimensional dynamic systems in which it is located. Although the trajectory (property) of each dynamic system is definitely measurable, the motion of multiple dynamic systems is not definitely measurable when combined. In other words, it is impossible to observe the direction and position and velocity of quantum motion at any given moment from outside the multiple dynamic systems. To measure or predict quantum motion from different dynamic systems, different results are bound to be obtained. Therefore, the uncertainty, randomness and superposition state of quantum motion are the essential properties of the operation law of multi-level and multi-dimensional dynamic systems. Except for particles or energieelements in high-energy radiation, everything in the universe exists and changes in motion in their own multiple dynamic systems. The motion of the energieelement described should be superimposed (iterated) along the motion of the multiple dynamic systems to reveal the true process. In fact, if you look at the motion of every person on Earth from outside the galaxy cluster, it would also take on a quantum state. Quantum effects are not understood because we now observe the motion of microscopic particles in multiple dynamic systems only from the four dimensions ( from the earth and its four dimensions), rather than from the iteration of multiple dynamic system. It is just as impossible to understand phenomena in three dimensions as it is to observe them only in two dimensions.

\section{B. Conjectures}

\section{Energieelements}

An energieelement is the most microscopic unit of human understanding of the universe and the most basic state of existence of cosmic energy and matter. It can be considered that a energieelement is the most basic microscopic particle constituting everything in the universe. The energy and mass of any matter are composed of two different forms of energieelements. Essentially, the mass of any object is the energieelement that makes up the object. The mass of an object on earth is measured or calculated in terms of its inertial mass, or gravity.

An energieelement is the smallest microscopic particle known and detected by instruments. It is a kind of microscopic particle in high speed motion and with very high energy. The energy of an energieelement is conserved and in a state of high-speed inertial motion.

\section{The rotational and inertial motion of a multi-level and multi-dimensional dynamic system}

The ordered motion of different quantities of energieelements forms different dynamic systems. Any dynamic system is an inertial system in rotational motion [1]. The power of any inertial motion system comes from the energieelements that make up the inertial system. At the most microscopic level, these inertial motion systems composed of different quantities of energieelements become the elementary particles of all classes. So far, scientists have identified and named 61 elementary particles [10]. An ordered inertial motion system formed by different numbers of protons, neutrons, electrons, etc., is a variety of atoms. An inertial motion system formed by one or more atoms becomes molecules. By analogy, from planets to stars, to galaxies and clusters of galaxies are inertial motion systems of different levels in rotational motion.

\section{Inertial forces are the source of power for everything in the universe}

Starting from energieelements, quanta, atoms, molecules, stars, star systems, galaxies and galaxy groups are all inertial motion systems of different levels composed of energieelements of high energy motion, and the inertial system of different levels has corresponding inertial forces. The strong and weak forces are the inertial forces (short-range forces) formed by microscopic particles; Electromagnetic force is the inertial force (long-range force) formed by the interaction between charged and magnetic objects. Gravity is the inertial force (remote force) formed by the inertial motion of macroscopic objects (stars and galaxies). Therefore, the four forces in the universe are essentially inertial forces, they are inertial forces of different levels of inertial motion systems. 


\section{Discussions}

\section{The origin of energieelements}

Everything in the universe is composed of energieelements, which is the smallest unit of matter (energy) that human beings can detect or confirm its existence by means of instruments and corresponding methods. What is the internal structure of a energieelement? Where did energieelements come from or how did energieelements come from? Further exploration of this problem is neither feasible nor necessary in terms of scientific and technological development and practical application. But it is certain that energieelements, like the universe, exist forever, have no beginning and no end, are eternal, and energy is also conserved.

\section{Infinity and infinitesimal}

The search for infinity and infinitesimal has never stopped, but it will never have an ultimate result. In this sense, infinity is a philosophical and mathematical concept that cannot be ultimate. In objective practice, the pursuit of ultimate infinity or infinitesimal is neither possible nor meaningful. If a theory is based on the ultimate infinity and infinitesimal it is impractical, it has no real material basis and no practical feasibility, it can only be a model of thought or it can only be an imaginary picture or a purely conjectural description.

\section{Absolute and relative space-time}

Absolute space without any energy or matter is meaningless and unthinkable. There is no such absolute space in the universe without any energy or matter.

The space with specific motion and change of matter and energy is the relative space of objective existence. The relative space of all things in the universe is manifested as the state and size of the existence or distribution of matter and energy, and is measured and expressed in three dimensions. Different motion of matter and energy forms different spatial characteristics.

The absolute time when no matter or energy exists and no movement changes is meaningless and inconceivable. Since there is no change in the motion of any material energy, there is no base or basis for measuring time. Colloquially, time cannot be measured without a frame of reference. So, there is no absolute time.

Time is based on the motion of specific matter and energy, it is relativistic. Relative time is defined as the motion and change process of specific matter and energy, which can be measured by period or frequency. Relative time can be measured and compared between different changes in the motion of matter and energy.

\section{Analyses}

\section{Chemical bond}

The atoms of the same multi-dimensional dynamical system interact with each other in the vortex motion, they are close to each other under the inertial forces of rotational motion, at the same time, the vortex energy quanta between them can also interact with electrons and other radioactive particles to create resistance to their proximity. The approaching force and the resistance force wind up to form a twisting force, which binds atoms to each other, and that's what bonds are all about.

\section{Dark matter and dark energy [7]}

When the temperature of the object drops to $0 \mathrm{~K}$, the energetic particles around the vortex, such as protons, neutrons, and atoms, are almost dispersed, and they will achieve a closer bond between them. At this time, the material density of the object will increase, and at the same time, the energy will hardly radiate outward, so their existence cannot be observed from a long distance. Dark matter is something that doesn't emit radiation, and it is also the way dark energy exists. The invisible quantum of energy in the universe is also how dark matter and dark energy exist when there is no radiation. All things in the universe operate in a multi-dimensional inertial system. The motion, change and interaction of all things in the universe are driven and maintained by different levels of inertial forces, and do not need dark matter or dark energy to drive or maintain.
[1] G. Yang, Energy and the Universe, Nature and Science, 457-460 (2019).

[2] G. Hao, (2020), https://www.netpad.net.cn/ resource_web/course/\#/217337.

[3] G. Hao, (2020), https://www.netpad.net.cn/ presentationEditor/presentationPlay.html\#218962.

[4] G. Hao, (2020), https://www.netpad.net.cn/ resource_web/course/\#/219053.

[5] B. Greene, The fabric of the cosmos: Space, time, and the texture of reality (Knopf, 2004).
[6] S. Hawking, The universe in a nutshell (Bantam, 2001).

[7] J. B. Kaler, Heaven's touch: from killer stars to the seeds of life, how we are connected to the universe, (Princeton University Press, 2009).

[8] P. C. D. Davies and P. Davies, God and the new physics, (Simon and Schuster, 1984).

[9] B. Clegg, The God effect: Quantum entanglement, science's strangest phenomenon, (Macmillan, 2006)

[10] B. Cox and J. Forshaw, The quantum universe:(and why anything that can happen, does), (Da Capo Press, 2012) 